\title{
ORGANIC RESIDUES FROM ULTRAVIOLET IRRADIATION OF INTERSTELLAR ICE ANALOGS
}

\author{
P. Modica ${ }^{1}$, P. de Marcellus ${ }^{1}$, D. Baklouti ${ }^{1}$, R. Brunetto ${ }^{1,2}$, M. Noun $^{3,4}$, \\ S. Della Negra ${ }^{3}$ and L. Le Sergeant d'Hendecourt ${ }^{1}$
}

\begin{abstract}
Interstellar ices are widely observed in the infrared spectra of regions where stars and planetary systems form. Photochemical and thermal evolution of these ices is simulated and studied in our laboratory where the resulting production of complex organic residues is routinely performed. Observations of their variability with slightly different starting chemical conditions can be performed by means of infrared spectroscopy. Organic residues have interesting chemical properties that make them good potential candidates for prebiotic chemistry. Numerous other analytical methods (GC-MS, HPLC, mass spectrometry, etc.) can be combined to refine the knowledge of their chemical composition and structure. We present here some results on the obtained organic residue resulting from subsequent heating and sublimation of the irradiated ices which show the formation of some macromolecular species.
\end{abstract}

\section{Introduction}

Organic compounds are abundantly present in extraterrestrial environments, like interstellar grains, comets, and asteroids. Interstellar grains present in dense molecular clouds accrete simple molecules and atoms from the gas phase forming an ice mantle (Sandford \& Allamandola 1993). As the material accrete on the surface, it is exposed to UV irradiation and/or cosmic rays (Prasad \& Tarafdar 1983; Tielens \& Charnley 1997). After energetic processing and thermal evolution,

\footnotetext{
1 Université Paris-Sud 11, Astrochimie et Origines, Institut d'Astrophysique Spatiale, UMR 8617, 91405 Orsay, France

2 CNRS, 9145 Orsay, France

3 Université Paris-Sud 11, IPNO, UMR 8608, 91405 Orsay, France

4 Lebanese Atomic energy Commission, National Council for Scientific Research, PO Box 11-8281, Beirut, Lebanon
} 
interstellar grains may result with a coating of a refractory residue of some hundredths of micrometers in size that contains many different very complex organic molecules (Greenberg \& Hage 1990). This material could have a fundamental role in the onset of molecular organic complexity so is important for further prebiotic chemistry (Le Sergeant d'Hendecourt 2011). Laboratory simulations do show the formation of such complex species from initially very simple ices after UV photolysis and warm-up to room temperature (Agarwal et al. 1985; Allamandola et al. 1988). Similar results can also be obtained from fast ion bombardment simulating cosmic ray irradiation (Moore 1983; Strazzulla \& Baratta 1991; Strazzulla et al. 2001).

\section{Laboratory experiments}

In laboratory we simulate some aspects of the astrophysical environments of interstellar/precometary ices. Basically we prepare room temperature gas mixtures of simple molecules $\left(\mathrm{H}_{2} \mathrm{O}, \mathrm{CH}_{3} \mathrm{OH}, \mathrm{NH}_{3}\right)$ that are known to be abundant in molecular clouds (Dartois 2006) and condense them under vacuum on a cold substrate at $80 \mathrm{~K}$ to simulate the accretion of molecules on dust grains. Simultaneously, we irradiate the solid sample by UV light simulating the radiation field in space. After that, the photolyzed sample is warmed-up until room temperature to simulate the thermal evolution of ices, for example in hot molecular cores. During the warm-up volatile species sublimate and a pale yellow colored material, stable at $300 \mathrm{~K}$, is left over: the organic residue. At the end of a cycle of one to three deposition/irradiation and warming-up, we recover the substrate and the sample that remains on its surface that will then be analyzed. IR spectrometry is used for in-situ monitoring of the samples during their preparation so that an IR spectrum of the residues is always obtained in this process. Detailed analysis is then performed by chemical analytical techniques such as GC-MS, mass spectrometry, and HPLC.

\section{Results}

The IR spectra of three different residues at room temperature are presented in Figure 1. The top panel shows a spectrum obtained from a $\mathrm{H}_{2} \mathrm{O}$ rich starting mixture $\left(\mathrm{H}_{2} \mathrm{O}: \mathrm{CH}_{3} \mathrm{OH}: \mathrm{NH}_{3}\right.$ in the ratio 10:1:1), the middle panel shows a residue spectrum obtained from a standard mixture $\left(\mathrm{H}_{2} \mathrm{O}: \mathrm{CH}_{3} \mathrm{OH}: \mathrm{NH}_{3}\right.$ in the ratio 2:1:1), and the bottom panel the residue spectrum of a standard mixture with methanol labeled with ${ }^{13} \mathrm{C}$ in order to exclude any biological contamination when traces amounts of specific molecules (e.g. amino acids) are searched for. All the spectra appear very similar, being dominated by the presence of a broad feature ranging from 3600 to $3000 \mathrm{~cm}^{-1}$ ascribed to carboxylic acids (-COOH), a strong double peaked band due to $\mathrm{CH}_{2}$ and $\mathrm{CH}_{3}$ in aliphatic chains, three strong features in the region from 1700 to $1500 \mathrm{~cm}^{-1}$ assigned to esters and amides, and numerous sharp features in the region of the fingerprints like the 1236 and $1007 \mathrm{~cm}^{-1}$ that are 


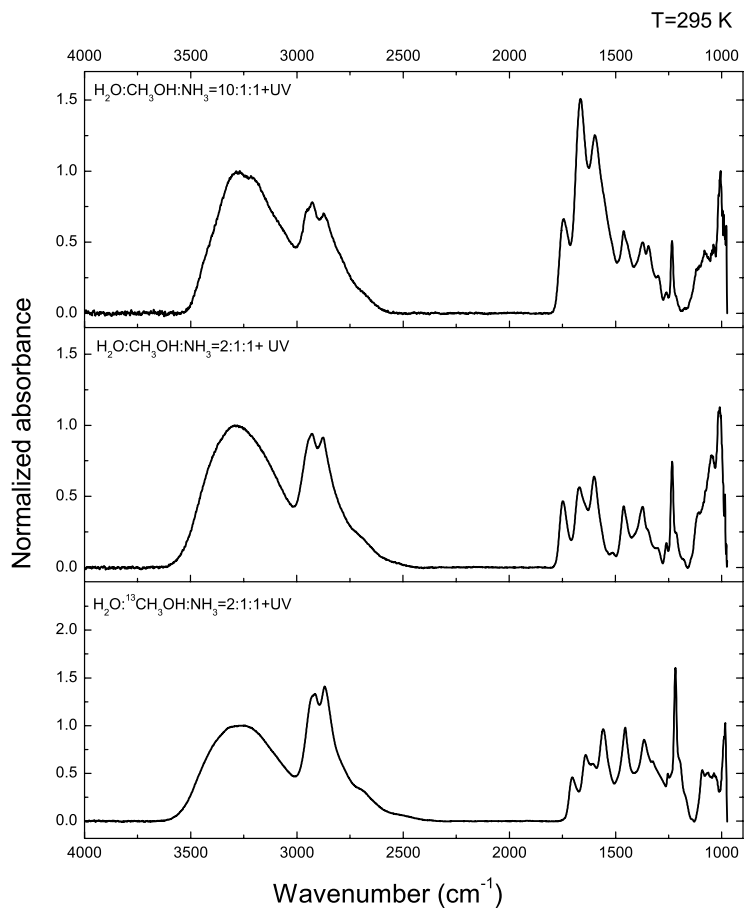

Fig. 1. IR normalized spectra of residues at $295 \mathrm{~K}$ obtained after UV photolysis of different ice mixtures.

Table 1. Infrared bands observed in organic residues at $295 \mathrm{~K}$.

\begin{tabular}{ccc}
\hline $\begin{array}{c}\text { Band position } \\
\mathrm{cm}^{-1}\end{array}$ & Assignment & Molecular function \\
\hline $3600-3000$ & $\mathrm{OH}, \mathrm{NH}$ str & alcohols, carboxylic acids, amines \\
$2957-2945$ & $\mathrm{NH}$ str & carbon chains \\
$2926-2923$ & $\mathrm{CH}_{3}$ str & HMT, aliphatic chains \\
$2875-2872$ & $\mathrm{CH}_{2}$ as str & HMT, NH $_{4}^{+}$, aliphatic chains \\
2150 & $\mathrm{CN} \mathrm{str}$ & nitriles? (after photolysis) \\
$1750-1745$ & $\mathrm{CO} \mathrm{str}$ & esters \\
$1675-1665$ & $\mathrm{CO} \mathrm{str}$ & amides \\
$1603-1599$ & $\mathrm{NH}{ }_{2}$ & amides \\
$1461-1458$ & $\mathrm{CH}$ def & $\mathrm{NH}_{4}{ }^{+}$, methyl groups, methylene \\
1370 & $\mathrm{CH}$ bend & HMT \\
$1235-1233$ & $\mathrm{CN} \mathrm{str}$ & HMT \\
1006 & $\mathrm{CN} \mathrm{str}$ & HMT \\
\hline
\end{tabular}

ascribed to hexamethylenetetramine (HMT, the most abundant and only detected molecule in the residue). Table 1 lists the bands along with their assignments and 
molecular functions (Muñoz Caro \& Schutte 2003; Muñoz Caro \& Dartois 2009; Bernstein et al. 1995). We note that changing slightly the initial composition of the mixture essentially affects some bands relative ratios, for example the $\mathrm{CO}$ group of esters versus the amides one.

Mass spectra of organic residues were obtained by laser desorption time-of-flight mass spectrometry (LD-TOFMS) at IAS. They reveal that residues are indeed complex organic mixtures with a wide mass distribution. In Figure 2 we show a spectrum in which macromolecular components having masses between 1000 and $3000 \mathrm{amu}$ are detected for the first time in a laboratory residue. The interest in these macromolecular components lies in their properties, such as their high chemical functionalization which results in their high water solubility that makes them good potential candidates for further prebiotic chemistry.

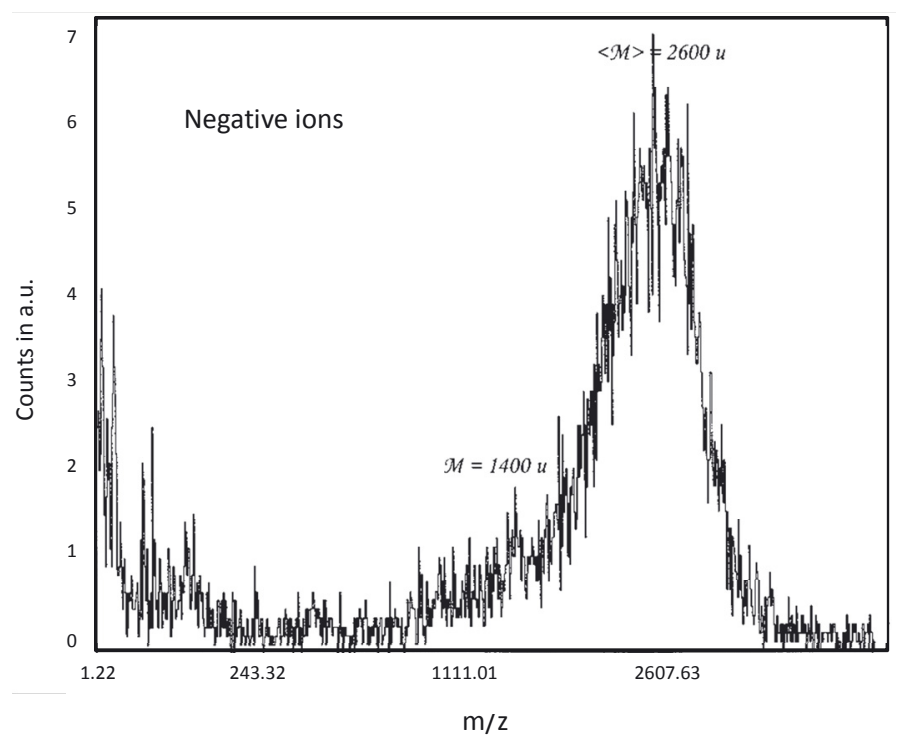

Fig. 2. Mass spectrum of an organic residue after laser desorption in negative ionization mode.

\section{Conclusions}

During the formation of planetary systems, grains accrete into small bodies such as comets and asteroids which capture the organic residue. Later they may bring it to planets and provide a source of prebiotic material on telluric planets such as the Earth (Oró 1961; Greenberg 1986). This material may be considered particularly suitable for further prebiotic activity if placed in an appropriate environment where liquid water may be present. Our experiments shed light on the composition of these potential prebiotic precursors and our simulations may provide a template for organic matter present in circumstellar discs as well as in meteoritic materials. 


\section{References}

Agarwal, V.K., Schutte, W., Greenberg, J.M., et al., 1985, Orig. Life Evol. Biosph., 16, 21

Allamandola, L.J., Sandford, S.A., \& Valero, G.J., 1988, Icarus, 76, 225

Bernstein, M.P., Sandford, S.A., Allamandola, L.J., et al., 1995, ApJ, 454, 327

Greenberg, J.M., 1986, in The Galaxy and the Solar System (Tucson: Univ. of Arizona Press), 103

Greenberg, J.M., \& Hage, J.I., 1990, ApJ, 361, 260

Dartois, E., 2006, A\&A, 445, 959

Le Sergeant d'Hendecourt, L., 2011, EPJ Web of Conferences, 18, 06001

Moore, H.M., Donn, B., Khanna, R., et al., 1983, Icarus, 388, 405

Muñoz Caro, G.M., \& Dartois, E., 2009, A\&A, 494, 109

Muñoz Caro, G.M., \& Schutte W.A., 2003, A\&A, 412, 121

Oró, J., 1961, Nature, 190, 389

Prasad, S.S., \& Tarafdar, S.P., 1983, ApJ, 267, 603

Sandford, S.A., \& Allamandola, L.J., 1993, ApJ, 417, 815

Strazzulla, G., \& Baratta, G.A., 1992, ApJ, 266, 434

Strazzulla, G., Baratta, G.A., \& Palumbo, M.E., 2001, Spectrochem. Acta part A, 57, 825

Tielens, A.G.G.M., \& Charnley, S.B., 1997, Orig. Life Evol. Biosph., 27, 23 